\title{
Ruido: intrusión sonora e intimidad acústica
}

ANA LIDIA M. DOMÍNGUEZ RUIZ, UNIVERSIDAD PEDAGÓGICA NACIONAL, MÉXICO

Fecha de recepción: septiembre de 2015

Fecha de aceptación: noviembre de 2015

\begin{abstract}
RESUMEN: Este artículo analiza el fenómeno del ruido a partir de la figura del intruso sonoro, es decir, un sonido extraño que invade ymodifica nuestro espacio privado. A través del concepto de intimidad acústica examinaremos tres dominios de la vida privada que requieren de condiciones de inmunidad sonora, es decir, estar a salvo del ruido: el descanso, el pudor y la abstracción. A partir de los testimonios de los habitantes de la Ciudad de México, recogidos de viva voz y en foros de Internet, daremos cuenta de las diversas maneras que tiene el ruido de trastocar los valores de la vida privada.
\end{abstract}

PALABRASCLAVE: sonido, ruido, intrusión, vida privada, México D. F.

\begin{abstract}
This article analyzes the phenomenon of noise based on the figure of the resounding intruder, which means a strange sound that invades and modifies our private space. Through the concept of "acoustic intimacy" we will examine three fields of the private life which require sound immunity that is being safe from noise: rest, decency and blockout. Based on testimonies of Mexico City inhabitants, taken orally and from internet forums, we will discuss several ways in which the noise disrupts the private life values.
\end{abstract}

KEYWORDS: sound, noise, intrusion, private life, México D. F. 


\section{INTRODUCCIÓN}

El espacio privado es el más personal de los espacios, aquél donde se recrea una atmósfera hecha al modo de quien lo habita; se trata de un lugar familiar, doméstico, reservado para el reposo y la secrecía. El lugar mejor identificado para la escenificación de la vida privada es la casa; sin embargo, existen otros lugares que cumplen esta función. El concepto francés chezsoi, queliteralmente significa "sentirse como en su propia casa", refiere justamente a la sensación de intimidad que se experimenta en un espacio cualquiera que se convierte en una zona segura y donde uno puede distenderse.

En el ánimo de proteger el dominio privado es imperativo mantener esta área intestina lejos de todo aquello que la ponga en peligro; es decir, de aquellas presencias que, al no estar invitadas a formar parte de nuestra intimidad, constituyan una amenaza. Esta sensación se sintetiza en la figura del intruso, una presencia que, dice Pascal Amphoux, "no puede penetrar más que por violación" (s/f, p. 39). Uno de los fenómenos de intrusión más comunes en las grandes ciudades es el ruido. Aquílo definimos comola personificación sonora de una presencia ajena que penetra un espacio privado y trastoca de diversas maneras la intimidad.

La intimidad acústica refiere a la sensación de seguridad experimentada en un espacio libre de intrusiones sonoras. Si bien es cierto que el ruido en las ciudades ha llegado a pensarse como algo hasta cierto punto inevitable, existen ciertas actividades cuya realización requiere de inmunidad acústica para poderse realizar. El trabajo que a continuación se presenta tiene como objetivos definir y caracterizar al intruso sonoro a través de un análisis de las diversas formas de injerencia del ruido en la privada de los habitantes de la Ciudad de México.

\section{EL INTRUSO SONORO}

La intrusión sonora es un fenómeno en cuya existencia intervienen criterios tanto objetivos como subjetivos. Objetivamente, la materialidad del sonido se asemeja a una suerte de fantasma que traspasa paredes, techos, puertas y ventanas, es decir, las fronteras que visualmente se han concebido para delimitar los espacios:

\footnotetext{
El sonido es un intruso por naturaleza ya que su comportamiento no obedece a la organización espacial a la que estamos acostumbrados y a partir de la cual solemos concebir la vida privada; es decir, aquella del sentido de la vista y el tacto cuya sustancia concreta les permite definir de manera mucho más clara un territorio. Pensemos esta diferencia, por ejemplo, a partir de las habitaciones delimitadas por muros que hacen evidente el adentro y el afuera, y donde el cierre y la apertura de puertas y ventanas constituyen un excelente mecanismo para controlar el acceso a la misma. El sonido, sin embargo, no reconoce a estas consistencias físicas como
} 
límites. El cuerpo mismo, en tanto territorio privado, no está capacitado para controlar la información que recibe a través de los oídos pues éstos, a diferencia de los ojos, no cuentan con párpados para escapar de los estímulos sonoros (Domínguez, 2011, p. 34).

Desde los criterios de la subjetividad -que es el aspecto que aquí interesa- la capacidad intrusiva de un sonido estará determinada por lo que cada quien perciba como incómodo o indeseable. Isabel López Barrio engloba las diversas sensaciones relativas a la inconveniencia de un sonido bajo la categoría de molestia, concepto que define como: "un sentimiento desagradable o una actitud negativa producida por un ruido no deseado o juzgado como innecesario en el espacio vital del individuo (...) o como un sentimiento displicente que surge al considerar que el ruido puede afectar negativamente la salud" (1997, s/n).

Desde esta perspectiva, la evaluación de un sonido variará de una persona a otra, incluso será cambiante para la misma persona, en circunstancias distintas. A partir de esta idea podemos comprender, por ejemplo, que una fiesta de vecinos nos moleste más un martes que un sábado o incluso que no nos moleste entre semana si es que estamos invitados a ella, o que se acepte con resignación el caos de la ciudad el lunes por la mañana pero no en domingo. La variabilidad sobre la percepción del ruido se explica por el hecho de que el sonido es un intruso sólo cuando desajusta nuestros hábitos o nos impide cumplir con nuestra disposición personal de actividades. Al respecto dice Bégout:

Eso que yo rechazo a veces con tanta violencia, no es el hecho de que el otro posea costumbres extrañas que me desagradan, que viva de manera diferente a la mía, que actúe de manera extraña e incluso repugnante. No. Lo que yo en verdad no puedo tolerar de la presencia de un extraño es la relativización desagradable de mis hábitos, de mis costumbres y de la organización de mi vida que él me obliga a realizar (2005, p. 439).

El intruso sonoro, al traer la incertidumbre del exterior, contribuye precisamente a producir esa sensación evocada por Bruce Bégout que casi todos hemos llegado a experimentar en algún momento: sentirse como extraño en su propia casa. Los sonidos capaces de hacernos sentir de esta manera son como las visitas que arriban a nuestra casa sin invitación, personas cuya aparición imprevista nos orilla a modificar nuestras costumbres, a generar ciertas reservas producto de la desconfianza; muchasveces son presenciasindeseables que seapoderan dealguna $\mathrm{u}$ otra manera de nuestro espacio y tienen, incluso, el atrevimiento de disponer sobre él.

Con la intención de observar la manera en que se comporta un intruso sonoro, hemos identificado tres áreas de acción de la vida privada que requieren de inmunidad acústica para poderse desarrollarse y cuyas funciones primordiales son afectadas por el ruido. Estos tres dominios de la vida cotidiana donde 
el ruido hace mayor merma pueden ser considerados, a su vez, como las tres maneras más recurrentes de violencia acústica indirecta experimentadas por los habitantes de esta ciudad.

\section{EL DESCANSO}

La interferencia del sueño es probablemente la función más perjudicada por el ruido, aquella cuya perturbación acarrea los mayores costos individuales y sociales, cuya violación desvirtúa más el sentido del habitar y una de las más reacias a ceder al proceso de habituación. Dicen que "A todo se acostumbra uno menos a no comer", a este dicho podríamos agregar que tampoco a no dormir, pues el sueño es tan importante para la vida comola comida; si no, preguntemos a cualquier persona qué tan dispuesta está a renunciar de manera frecuente y prolongada a sus horas de descanso, y a un médico cuánto tiempo puede pasar alguien sin dormir antes de caer en un estado de shock.

El descanso se guarda como un tesoro, hay quienes se dicen dispuestos a soportar el caos de la ciudad con tal de mantener bajo resguardo la tranquilidad de su espacio privado. Las horas de sueño no se negocian y pocas veces se ceden; he aquía un ciudadano desesperado antela dificultad que tiene para conciliar el sueño por culpa del ruido: "Las personas que vivimos cerca de un antro vemos perturbado nuestro sueño y por ende nuestra calidad de vida se ve afectada, no sólo por el ruido de la música sino también por el de los clientes alcoholizados y escandalosos. ¡Por favor, déjennos dormir!” (Eloísa, en Ciudadanos en red, 2010, s/n).

Dormir a medias, despertarse varias veces a lo largo de la noche y no cumplir con los horarios de descanso son algunas de las protestas de los habitantes de Ciudad de México. Consideremos algunos testimonios de la manera en la que diversas actividades violentan los periodos de descanso; en ellos descubriremos que, para quien pretende cumplir su ciclo de descanso, no hay enemigo pequeño:

Los camiones que van de Coacalco a Indios Verdes en cada esquina tocan el claxon muy escandalosamente y muy repetitivo. No les interesa la hora, así sean las 4 de la mañana, durante el día y hasta la noche, a tal grado que las personas no pueden terminar su sueño completamente por este espantoso ruido (en Ciudadanos en red, 2008, s/n).

Vivo en un edificio de departamentos en la colonia Mixcoac. Mi problema es que los vecinos que viven justo arriba de mí son muy ruidosos; a lo largo del día golpean y arrastran objetos en el suelo, que es mi techo, y el ruido nos llega muy fuerte. Pero lo peor es que durante la noche hacen los mismos ruidos o incluso más fuertes hasta la una o dos de la madrugada" (en Buzón Prosoc, s/f, s/n).

Es una pareja de desgraciados (una mujer y un hombre). Los desgraciados se meten juntos a la ducha a las 7 de la mañana y se ponen a cantar canciones tan feas y corrientes, con una voz de corta-venas. ¡Porfas ya cállense y dejen dormir! (en Answers Yahoo, s/f, s/n). 
El siguiente caso apareció en un foro de discusión sobre ruido en la página de Answers Yahoo, al que se acercó una persona para pedir consejo de alguien que hubiera pasado por una situación similar. El detalle del relato y la atención puesta sobre el desarrollo de la actividad turbadora durante toda la noche revelan el grado de ofuscación del narrador por no poder dormir y por no poder hacer algo para remediar su desesperada situación. Su pregunta inicial, y con la cual se abrrió el foro fue: “¿Se puede hacer algo contra las fiestas delos vecinos?”. El caso es el siguiente:

\footnotetext{
Hola chicos. Miren, la verdad yo no tengo nada en contra de una fiesta de vez en cuando, pero estos tipos se agarran tres veces por semana. Justo hace cinco minutos empezaron. Son las 12 de la noche. Vibra toda la casa... Son casi las 3 de la mañana. Estoy bien cansada, desvelada. Ayer tampoco me dejaron dormir. Oigan, ¿les puedo enviar a la policía? Es que de verdad, tres días seguidos lo mismo... Son casi las 4 de la mañana. Salí en el auto a buscar la dichosa casa (mi fiel perrita me acompañó). Resulta que está por lo menos a cuatro cuadras de mi casa y para colmo en el fraccionamiento vecino. Los vigilantes de la entrada no quisieron dejarme pasar ni darme datos de la dirección... En este ratito entraron como veinte autos que iban a la fiesta, así que calculo ha de ser una fiesta como de cien o doscientos invitados. ¿Alguna sugerencia para esas ratillas? (en Answers Yahoo, $2009, \mathrm{~s} / \mathrm{n})$.
}

La llamada vida nocturna de la ciudad (fiestas privadas, de salón y a la actividad de establecimientos mercantiles tales como bares, discotecas y restaurantes) es una de las razones que más perjudican los periodos de descanso. Son innumerables las quejas por emisiones sonoras causadas por el parloteo de los usuarios y por equipos de sonido, karaokes, altavoces y discomóviles que, lanzados al espacio público, impiden a los vecinos de estos lugares conciliar el sueño, particularmente durante los fines de semana.

No sólo los que viven un rutina "normal" sufren los embates de la actividad nocturna, también quienes tienen una rutina inversa y viven de noche reportan problemas para dormir. Los trasnochados dicen ver perturbadas sus horas de descanso a causa de la actividad diurna; una chica narra la molestia que le provoca el trajín diurno:

Nosotros hacemos fiestas los viernes y los sábados, a veces también entre semana. A veces nos dormimos a las siete de la mañana. Bueno, eso de dormir es un decir, porque como a las ocho comienzan las actividades en el parque que está aquí junto, llegan los boyscouts, la gente que va a hacer ejercicio, pasa el gas. Y la verdad es muy molesto tratar de dormir con tanto ruido (Elena, entrevista, 2012).

Independientemente de cómo se decida construir la rutina, para todos es necesario cumplir con el equilibrio vital que ayuda a sostener el respeto por las horas de sueño. Dice Gastón Bachelard que para el hombre la vida comienza durmiendo bien, reconociendo con esto el valor primordial del descanso, y 
agrega: "una función no puede ser permanente, es necesario que le suceda un periodo de no-funcionamiento desde que la energía disminuye y hasta que ésta se desgasta" (1993, p. 23). Respetar esta máxima permite la renovación y el dispendio diario de energía; su transgresión no sólo pone en riesgo la seguridad y la confianza de los espacios privados, sino que atenta contra el equilibrio vital que mantienen los periodos de sueño y vigilia.

Pedro Barruecos, jefe de Audiología y Foniatría del Hospital General de México, explica que el ruido perjudica directamente los ciclos de actividad y sueño de una persona durante veinticuatro horas, lo que modificalas funciones de sus sistemas cardiovascular, nervioso y endocrinológico, llegando incluso a provocar cambios en la composición de la química sanguínea; y agrega:

Basta con imaginar a una persona que duerme de las 12:00 a las 06:00 horas, pero despierta tres horas antes interrumpido por el ruido de un bar que está frente a su casa.

$\mathrm{Al}$ amanecer no presentará pérdida auditiva, pero se dirigirá al trabajo cansado, estresado, rabioso, impotente, desesperado. En los meses siguientes su rendimiento disminuirá y se alterará su ritmo respiratorio, cardiaco, digestivo (Barruecos, 2009, s/n).

Leamos otros dos testimonios que narran los perjuicios ocasionados por el mal dormir a causa del ruido nocturno:

Fueron siete u ocho meses de dormir mal o no dormir por el desmadre de Ali. Andaba de pésimo humor, a donde quiera que iba cumplía mis obligaciones pero de mal humor. Te pesan los ojos, te duele el cuerpo. Me dormía en las tardes. Dejé de hacer muchas cosas: no pintaba, no podía hacer ejercicio. Te enojas con mucha facilidad, te desquitas con el de junto, con quien más confianza tienes (Bernardo, entrevista, 2008).

Yo vivo en las calles de Cihuacóatl y Chiconautla y el ruido que se genera aquí es por vecinos que llevan en el más alto volumen el estéreo de su auto, no importando si es de día o de noche: jóvenes que se pasean en motos, perros que son hacinados en las azoteas y ladran en exceso por las noches, y ni qué decir de los vendedores que a claxonazos o gritos vienen a ofrecer sus mercancías. Total que esto parece no ser de importancia hasta que de la nada tienes un carácter irritable, distraído y con un cansancio extremo (en Ciudadanos en red, 2009, s/n).

Diversos estudios dedicados al análisis de los efectos a largo plazo por la exposición al ruido nocturno han ayudado a comprobar quelos sujetos expuestos a elevados niveles de ruido, en comparación con los de áreas silenciosas, se muestran más ansiosos, nerviosos y cansados durante el día. De acuerdo con las Guías para el Ruido Urbano $(1995,1996)$ publicadas por la Organización Mundial de la Salud, los trastornos provocados por el ruido son de dos tipos: los primarios, resultantes de la dificultad para conciliar el sueño o de su interrupción, tienen como consecuencia cambios en la presión arterial, arritmia cardiaca, vasoconstricción, variación en el ritmo respiratorio y sobresaltos cor- 
porales; los trastorno secundarios, cuya particularidad es que no se presentan en el momento sino un día después e incluso pueden prolongarse por varios días; son fatiga, depresión, irritación, angustia, ansiedad, dolores de cabeza, reducción del rendimiento y sentimientos de impotencia.

La alteración de esta vital función del espacio privado obliga a los habitantes de la ciudad a modificar sus costumbres durmientes, tal y comolo apuntaba Bruce Bégout al hablar de la relativización de hábitos que el intruso obliga a realizar. Así, al preguntar a varios usuarios del Metro la razón por la cual utilizaban este medio de transporte para dormir, muchos respondieron que era a causa de no poder cumplir con sus horas de sueño en casa por la presencia del ruido, aunque también declararon razones como la fatiga normal del día a día o la extensión de los trayectos que impone una siesta. Incluso ahí, en ese remanso público de descanso, el intruso sonoro perturba el sueño: "Son insoportables los vagoneros, son de lo peor que hay en el Metro. Habemos personas que, como yo, intentamos dormitar en el trayecto de uso del metro y estas personas, los vagoneros, no nos dejan, son molestos e irritantes" (en Ciudadanos en red, 2009, s/n).

Otras modificaciones a la rutina originadas por el ruido son los cambios de los horarios con la intención de que éstos se acoplen al tiempo del intruso, el uso de tapones para los oídos o el implemento de máscaras sonoras, es decir, sonidos que uno mismo programa con la intención de que cubran los ruidos exteriores, tales como audífonos, ambientes musicales o "el ventilador que una vecina prendía a la hora de dormir para no escuchar el ruido del bar de enfrente".

\section{LAABSTRACCIÓN}

Otra de las funciones del espacio privado es brindar al individuo la oportunidad de poder estar a solas consigo mismo, de recogerse física y mentalmente. La abstracción es una actividad que requiere del ensimismamiento, entendido como la entrega del hombre a sus propios pensamientos. El espacio propicio donde se concreta dicho proceso es la mente, un espacio primigenio donde se crea y se recrea el yo. La mente es el lugar más recóndito del mundo y aquella instancia última a la que se recurre para buscar seguridad; en los casos de reclusión, por ejemplo, a esto se reduce la privacidad de una persona: más allá de su pensamiento todo es exterior, y todo aquello que ocurre en su cabeza es su último resquicio de intimidad.

Son muchos los testimonios que dan cuenta de la manera en que el intruso sonoro impide a los habitantes de la ciudad trabajar, concentrarse, encontrar remansos de tranquilidad o espacios de aislamiento que le procuren momentos de soledad. La ciudad pareciera ejercer una especie de acoso al negarse a abandonar al urbanita incluso ahí en su terreno más íntimo: el pensamiento. 
Al respecto de los inconvenientes del ruido, decía Schopenhauer: "El ruido es una tortura para los intelectuales y la más impertinente delas perturbaciones (...) la cantidad de ruido que uno puede soportar sin que le moleste, está en proporción inversa a su capacidad mental" (en Baigorri, s/f, p. 3).

La historia relacionada con el ruido nos muestra múltiples anécdotas que manifiestan las trabas que el intruso sonoro impone al trabajo intelectual. Prestemos atención al siguiente fragmento de la epístola que, en este tenor, Séneca le dirige a Lucilio alrededor del año 40 a.C.:

\footnotetext{
Moriré si el silencio es tan necesario como parece para el que quiere retirarse al estudio. Y así me hallo rodeado de un griterío abigarrado: habito sobre unos baños. Figúrate todos los tipos de gritos que pueden repugnar a los oídos" (Juristas contra el ruido, s/f, s/n).
}

Muchas de las quejas alzadas durante la Revolución Industrial manifiestan la imposibilidad de los trabajadores de gabinete-doctores, abogados y artistas, por mencionar algunos- para realizar su trabajo a causa del ruido. Hoy en día las condiciones no han cambiado. Recientemente un profesor universitario se quejaba de no poder trabajar en su oficina a causa de las actividades estudiantiles:

\footnotetext{
Los estudiantes disponen por completo de la universidad: ponen música en el patio, dirigen discursos con micrófonos y bocinas. Es increíble la falta de respeto a nuestro trabajo como profesores, ni siquiera puedo trabajar en mi cubículo, no puedo leer ni preparar clases en mi espacio de trabajo (Rodrigo, entrevista, 2011).
}

Concebir, planear, ponderar, especular, imaginar, analizar y juzgar son algunos de los procesos que intervienen en la actividad intelectual y cuya condición primigenia es el aislamiento; idealmente el gabinete, por ser un espacio privado, procura a sus ocupantes esa atmósfera personal y controlada necesaria para poder abstraerse del entorno y dar rienda suelta a la vida interior. En esta búsqueda se suele pensar en el silencio como una delas condiciones fundamentales para alcanzar la abstracción; el silencio, dice David Le Breton, contribuye a la creación de espacios y momentos propicios para el recogimiento. Y agrega:

El silencio instala en el mundo una dimensión propia (...) el tiempo transcurre sin prisa, al paso que marca el ser humano, invitando al reposo, a la meditación, a la introspección. Estos lugares embutidos de silencio se separan del paisaje, y resultan propicios para el recogimiento personal (2006, p. 114).

Los espacios silenciosos promueven la inspiración, la meditación y el descanso. A esto se debe que el silencio sea un requisito fundamental en las salas de estudio, las bibliotecas, las iglesias y los hospitales; lugares que por motivos diversos precisan de condiciones de mutismo y reserva para que sus ocupantes pueden "sumergirse en sus pensamientos", "perderse en la lectura" o "ser 
presos de la ensoñación”. Son precisamente estos estados de absorción los que destruye el ruido, ya sea impidiendo su alcance o sacándonos de ahí. Quien accidentalmente hace ruido en una biblioteca y pide disculpas por ello, es porque se ha dado cuenta de que quienes leen no están ahí y que su irrupción los ha regresado de aquel lejano lugar.

El silencio -al contrario de lo que suele pensarse- no es la ausencia de sonido, sino una condición de relajación sonora debida a la disminución de los estímulos, al alejamiento de la fuente o a la reducción del volumen. Si bien el grado cero de la sonoridad no existe como estado natural, la atenuación puede llegar a ser tal que podemos escuchar esas voces, que suelen estar ocultas bajo el ruido, entre las que se cuenta, incluso, nuestra propia voz interior. En la ciudad, particularmente en la de México, sin embargo, es muy difícil encontrar lugares que propicien de manera natural estos estados, sólo algunas veces en la noche o ciertos días del año, o en espacios muy interiores o muy alejados. En general, estas actividades se desarrollan en medio del bullicio urbano, incluso aquellas que ocurren en recintos planeados para dichas funciones, e implican un gran dispendio de energía en tanto que suponen una lucha contra la misma ciudad, tal y como acontece en las siguientes narraciones:

Me desconcentraba de la lectura, lo que hago mucho al viajar es que siempre estoy como con algo y voy leyendo, entonces como que tienes tus cálculos mentales del tiempo de las estaciones y te concentras en la página y, de repente, que cada tanto pase un vendedor con un volumen realmente molesto, te distrae (Iván, entrevista, 2010).

Siviajas en transporte público es muy cotidiano encontrar a los vendedores, pero no siempre vas como preocupado por tu recorrido y estás pensando en otras cosas y estás evitando el contacto visual, y, en cuanto llegan éstos, te sacan de tu pensamiento e inevitablemente tienes que ponerles atención (Mirna, entrevista, 2010).

En años recientes la presencia de ruido en la escuela se ha convertido en una preocupación, pues sus costos pesan justamente sobre el rendimiento escolar. El ruido en los salones de clases, tanto del que proviene del exterior como el de los salones contiguos e incluso el que se produce en la misma clase, constituye un obstáculo para la concentración, ocasiona la pérdida de atención, dificulta el cálculo y entorpece el proceso de lectoescritura:

En la Avenida 553, a espaldas de la Secundaria Técnica núm. 41 Alfonso Sierra Partida, se estableció un vendedor ambulante que expende jugos de frutas. Ahora ha expandido su ilegal negocio a la venta de discos, por lo que para llamar la atención opera un aparato de sonido el cual pone a todo volumen, ocasionando con ello que los maestros y alumnos de la secundaria pierdan la concentración. Por nuestra parte, en mi casa somos personal docente y requerimos de un mínimo de silencio para preparar nuestras clases y continuar nuestra actividad de investigación (en Ciudadanos en red, 2009, s/n). 
De acuerdo con Evans (1990), la exposición continua a elevados niveles de ruido interfiere en la percepción del habla e incide de manera negativa en las adquisiciones relacionadas con la alocución, el dominio de la lengua escrita y con todos los aprendizajes vinculados a los mismos.

\begin{abstract}
Así, los resultados de diferentes investigaciones planteadas en relación a este tema, (Moch, 1984; Hygge, 1993) coinciden al señalar que, cuando los niños, por razón de la ubicación de sus hogares y/o de la escuela, se encuentran sometidos de manera continuada al ruido, éstos muestran una capacidad de atención y de discriminación auditiva significativamente menor en comparación con niños no expuestos al ruido, incrementándose este efecto con el tiempo de exposición: a mayor tiempo de exposición, mayores rasgos de distracción y menor capacidad de atención (López Barrio, 1997, s/n).
\end{abstract}

\title{
ELPUDOR
}

El pudor es una sensación de vergüenza y recato que se experimenta como protección a la intimidad; en tanto territorio privado es también un área violentada por el ruido, particularmente en lo que se refiere a la escucha de escenas sexuales. Al respecto, Simmel comenta: "Toda convivencia estrecha descansa en el hecho de que, merced a hipótesis psicológicas, cada cual sabe del otro más de lo que le muestra de un modo inmediato y voluntario" (1977, p. 653). Es así que el oído nos permite acceder secretamente a la privacidad de las habitaciones, a aquellos lugares donde el vecino, al no ser consciente de que se le escucha y al no reparar en el desamparo acústico de su casa, no tiene reserva alguna en relación con lo que dice y hace:

\footnotetext{
Vivo en una casa donde las paredes son compartidas con las del vecino. Mi recámara está pared con pared y son un matrimonio muy inmoral. Ella grita peor que cualquier película porno y se escucha exageradamente en toda la calle, pero, obvio, más en mi casa. Y es muy molesto e incómodo porque uno "trata" de dormir y cuando ya agarramos el sueño profundo empiezan a altas horas de la noche o muy temprano a las 5 de la mañana con sus cabecerazos y los gritos exagerados de ella (en Answers Yahoo, 2010, s/n).
}

Esta sigilosa intromisión en territorios íntimos tiene dos efectos: por un lado alienta la curiosidad del escucha, pues si bien en una primera instancia no es nuestra intención conocer esos episodios, resulta muy fácil engancharse con las historias y convertirse en ávidos escuchas; por otro lado, la falta de intimidad acústica produce incomodidad a causa de saber del otro, mucho más de lo quisiéramos. Ésta es precisamente la razón del malestar que aparece en numerosos testimonios de quienes narran haber escuchado a sus vecinos mantener relaciones sexuales o discusiones violentas. 
Pongamos atención a la siguiente discusión aparecida en Answers Yahoo y que fue abierta por una persona que pide ayuda para poder lidiar con la intimidad de sus vecinos:

Les cuento que me mudé a una vivienda Infonavit y desde el primer día se empezaron a escuchar sus descaradeces; puse un letrero afuera de su cuarto, que está pegado al mío, diciendo "Más discreción” y, al parecer, le subieron el volumen por las noches. Todas las noches es lo mismo, ambos se dicen sus cosas sucias y hasta ya aprendí su abecedario en inglés.

A esta petición de ayuda una mujer le contesta:
Te entiendo bastante bien, a mí me pasa igual. Primero tuve unos vecinos que decían vulgaridad y media, y yo hasta me ponía a llorar porque mis bebés estaban descansando y despertaban asustados. Es una vecina que vive sola pero no sé ni a qué horas mete al fulano, pero gime y gime tan fuerte que ofende a cualquiera, además no creo que cada vez que tenga una relación sexual -todas las veces- sea tan buena como para gemir así.

Roland Barthes hace una breve pero sustanciosa reflexión sobre el papel de la escucha en el vivir-juntos, particularmente refiere a la relación entre ésta y la sexualidad a través de un mecanismo de velada complicidad con el prójimo -el otro próximo- que denomina escucha espía, y que consiste en escuchar al otro mientras mantiene relaciones sexuales en el cuarto contiguo: "La pared, límite de la respetabilidad, máscara puesta a la vista, es forzada por la escucha" (2003, p. 132). Al respecto, David Toop ha analizado el fenómeno de la escucha furtiva a partir de una serie de pinturas de Nicolaes Maes (1634-1693), pintor holandés discípulo de Rembrandt, titulada “The eavesdropper” o el fisgón. Se trata de una serie de escenas donde Maes presenta a un voyeur sonoro que, escondido detrás de una pared o una puerta, accede veladamente a una escena que se desarrolla en supuesto secreto; son escenas de una vida privada ajena que pueden ser escuchadas o vistas, y en donde puede o no mediar la voluntad.

\footnotetext{
La forma más seria de la escucha furtiva, aquella en la que se descubre a través de una escucha sigilosa un tipo de información íntima que antes se guardaba con reserva, compromete al oyente en una confusa mezcla de ambivalencia y culpa. (...) Durante los episodios de este tipo, he experimentado la intensa sensación, casi alucinatoria, de estar involucrado en espacios distintitos únicamente conectados por el espacio auditivo que fluye entre uno y otro (Toop, 2013, p. 125).
}

Más que el acto intrusivo, lo que molesta o incomoda de estas escenas es el tipo de información al que el escucha accede sin desearlo o al que es introducido sin mediación de su voluntad, y que lo coloca en una posición de íntima proximidad con el otro, una familiaridad que no ha construido y que provoca su vergüenza. 


\section{CONCLUSIÓN}

Mediante el análisis de las diversas formas que tiene el ruido de desestabilizar las funciones del espacio privado, pretendimos conocer las implicaciones de este fenómeno y llevarlo más allá del ámbito de lo físico, que es donde comúnmente se le sitúa. Cuando ubicamos al ruido en el marco de las prácticas sociales, el enfoque acústico resulta insuficiente en tanto que explica tan sólo uno de los aspectos que conforman su compleja naturaleza. Hemos visto que en la construcción de la categoría de ruido intervienen factores de tipo físico, anímico, psíquico y social. Un determinado sonido molesta porque invade un espacio que se considera privado, ya sea que se trate de nuestra casa, nuestro cuerpo, nuestro coche, o de entidades menos concretas que representan los valores de intimidad como la rutina, el confort, el pudor, la tranquilidad o los momentos de trabajo y concentración.

Es precisamente esta capacidad de intromisión y esta cualidad de no invitado lo que convierte a un sonido cualquiera en ruido, es decir, un intruso sonoro que traspasa loslímites de nuestra privacidad, se impone sobre nuestra disposición del mundo y amenaza el equilibrio de nuestra esfera privada produciendo sentimientos de incomodidad y ansiedad. El presente trabajo se encaminó a analizar la naturaleza agresiva del ruido desde una perspectiva que nada tiene que ver con la potencia sonora.

El fenómeno de intrusión puede, así, definirse como una forma de violencia acústica que se ejerce mediante imposición. El ruido aparece como forma de dominación, es decir, una manifestación concreta del poder que se revela como una imposición de voluntades a través de diversas prácticas dominatrices del espacio sonoro y que nos obligan a escuchar lo que no queremos, y cuya capacidad perturbadora acarrea serios costos en la salud pública, física y social de los habitantes de las ciudades. 


\section{REFERENCIAS:}

Amphoux, P. (s/f). Le chez-soi dans tous les sens. Arch \& comport, vol. 5, 2, pp. 135-150.

Bachelard, G. (2002). La poética del espacio. México: Fondo de Cultura Económica.

Baigorri, A. (s/f). “Apuntes para una sociología del ruido”, disponible en http://www. insumisos.com/bibliotecanew/Sociologia\%20del\%20ruido.pdf

Barthes, R. (2003). Cómo vivir juntos. Argentina: Siglo XXI.

Bégout, B. (2005). La découverte du quotidien. Paris: Allia.

Domínguez, A. (2011). Digresión sobre el espacio sonoro. En torno a la naturaleza intrusiva del ruido. Cuadernos de vivienda y urbanismo, vol. 4, 7, Instituto de Vivienda y Urbanismo, Pontificia Universidad Javeriana, pp. 26-36.

El Universal del Valle (22 de diciembre de 2009). Violan norma de ruido antros y bares de la delegación Benito Juárez. Recuperado de: http://www.eluniversaldelvalle. $\mathrm{mx} /$ detalle1292.html

Juristas contra el ruido. El ruido en la antigua Roma. Recuperado de: $<$ http://www. juristas-ruidos.org/Documentacion/Epigrama_Marcial.pdf $>$ S/f.

Le Breton, D. (2006). El silencio. Madrid: Sequitur.

López Barrio, I. (1997). Medio ambiente y salud. Impacto del ruido, en Papeles del psicólogo, núm. 67, España.

Simmel, G. (1977). Sociología. Estudios sobre las formas de socialización. Revista de Occidente, Madrid.

Toop, D. (2010). Resonancia siniestra. Madrid: Caja Negra Editora.

\section{ANA LIDIA M. DOMIINGUEZ RUIZ}

Docente-investigadora de la Universidad Pedagógica Nacional, México. Miembro del Sistema Nacional de Investigadores y doctora en Ciencias Antropológicas por la UAM-Iztapalapa. Es autora del libro La sonoridad de la cultura. Cholula: una experiencia sonora de la ciudad y de varios trabajos sobre socioantropología del sonido, violencia acústica y educación sensorial. Es miembro del equipo de investigación del "Sonidos en el Aula», proyecto financiado por el CONACULTA y el ISSSTE.

E-mail:unalaid@hotmail.com 